\title{
A New Design for the BirthSIM Simulator to improve realism
}

\author{
Nicolas Herzig, Richard Moreau and Tanneguy Redarce
}

\begin{abstract}
This paper presents a new design of the BirthSIM simulator. Its goal is to help obstetricians and midwives to train and improve their skills during childbirth delivery. The new version of the BirthSIM is more actuated than the previous version in order to be more biofidelic and cover various scenarios. The direct and inverse geometric models of the haptic interface are presented. The working space reached by the fetal head is computed and validates the proposed design which allows to reproduce all fetal head trajectories inside the pelvis. The novelty is illustrated by an example which presents a simulated trajectory stemmed from a sacrum shape measure. The inverse geometric model allows to compute the actuators displacements and thus to validate the chosen components.
\end{abstract}

\section{INTRODUCTION}

Nowadays, medical simulators are more and more used in medical education. Medical simulators are available in different fields of surgery such as laparoscopy and Mini Invasive Surgery, anesthesia or obstetrical.

During the past few years, Ampère laboratory developed a childbirth simulator called BirthSIM [1]. It has been designed especially to train and evaluate the instrumental delivery gestures. It consists of anthropomorphic models of maternal pelvis and fetal head. Obstetrics forceps are instrumented to measure their displacements [2]. Several scenarios have been implemented on the BirthSIM simulator.

Trainees can thus proceed to a risk free training on forceps blade placement and forceps extraction. A visualization interface is also available to let the trainee to see inside the pelvis and improve his gestures while using forceps. The BirthSIM simulator has currently only 3 degrees of freedom (DOF). The first one is an actuated translation driven by a pneumatic actuator which allows to simulate linear displacement inside the maternal pelvis and the efforts involved during deliveries. The two others are passive rotations to place the fetal head in an initial desired orientation.

Several obstetrical simulators are available with different features. For instance Lapeer et al. chose to develop an augmented environment to simulate obstetric forceps delivery [3]. This simulator offers users to visualize their instruments inside the maternal pelvis. There is however no haptic interface to train to forceps extraction. Kim et al. has developed another simulator [4], it was designed in order to compare different delivery process. They chose to simulate the uterine contraction by inflating an airbag. This simulator is more biofidelic than the BirthSIM simulator, but the fetal head is not controlled so it does not offer a good repeatability

N. Herzig, R. Moreau and H. T. Redarce are with the Laboratoire Ampère, UMR CNRS 5005, Université de Lyon, INSA-Lyon, F-69621 Villeurbanne Cedex, France (e-mail: nicolas.herzig@insa-lyon.fr, richard.moreau@insalyon.fr, tanneguy.redarce@insa-lyon.fr). of scenarios. Sielhorst et al. [5] and Abate et al. [6] have worked on delivery simulators coupling augmented reality and haptic interface. The first one is based on a 6 DOF industrial robot to control the head trajectory. This kind of robots is easy to use but the range of the efforts given by the robot is oversized. The second one use an industrial exoskeleton haptic device. This device coupled with virtual reality goggles allows a perfect immersion of user and a good haptic feedback, but it is not appropriate for a team work practice. Some simulators are commercially available and one of the most advanced is the NOELLE simulator [7]. It is dedicated to team training but it is not adapted to evaluate specific gestures of a practitioner.

According to obstetricians remarks and compared to the literature, the BirthSIM simulator needs several improvements. For instance, scenarios for team training should be integrated moreover the simulator has to be more biofidelic. The aim of this paper is to focus on this last point. As previously said, the current BirthSIM simulator has only one actuated DOF. In order to improve the simulator anthropomorphism and in particular to respect the physiology of a childbirth, the number of DOF has to be increased and the haptic feedback has to be improved. This paper is divided into three sections. Section II introduced the study of the fetal head trajectory during its progress inside the maternal pelvis. Section III is dedicated to the design and the model of the new version of the BirthSIM simulator. Section IV deals with a simulation to validate the model and the design. We then conclude by discussing on the contributions and our future experimental plans.

\section{COMPuted TRAJectory}

To improve the realism of the simulator, the physiology of a childbirth has to be respected. The main drawback of the BirthSIM simulator is due to the only one actuated DOF. It is not enough to correctly reproduce the fetal head trajectory during the delivery. Indeed the fetal head has a curve trajectory which follows the sacral curvature of the pelvis. This trajectory is illustrated in Fig. 1. In order to simulate the fetal head movement inside the maternal pelvis, four DOF are necessary: two translations to reproduce this trajectory and two rotations to simulate the bending and the rotation of the fetal head.

One of the difficulty in medicine is to obtain in vivo data. One solution is to use numerical models which allow to proceed to a numerical childbirth delivery simulation. The aim of the SAGA project in which we are involved is to develop such models ( $c f$. acknowledgement section for details). These models interact with each others and take into accounts their 


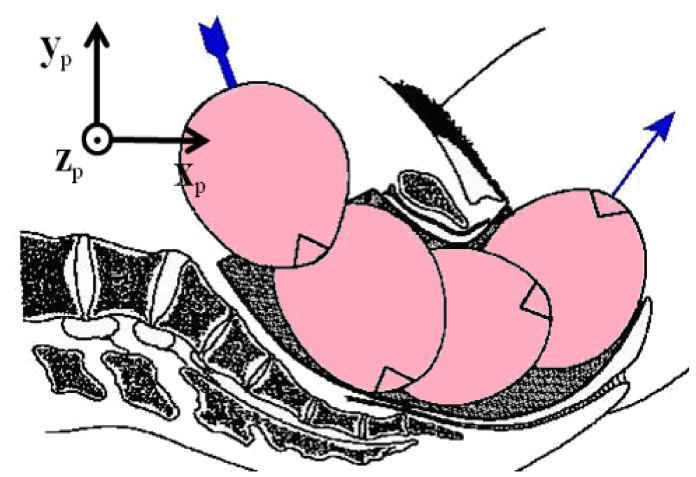

Fig. 1: Fetal head trajectory in the maternal pelvis [8]

deformations in order to simulate a childbirth. This numerical simulation allows thus to obtain a reference trajectory for the fetal head during its progress inside the maternal pelvis. Indeed, the ultimate goal of the SAGA project is to link the haptic interface with this numerical simulation and exchange data to improve the training possibilities of the BirthSIM simulator.

Buttin et al. already proposed a numerical simulation of a childbirth using finite element models [9]. In this work, some forces, corresponding to the uterine contractions, are applied on the numerical models. It is thus possible to simulate a childbirth and in particular to calculate the fetal head trajectory. As their models are deformable, contrary to the manikins used in the BirthSIM simulator, the center of the fetal skull, where the deformations are lower, has been tracked. This point has been tracked from the beginning of the delivery to the position where the head started to get out of the pelvis. In order to check this trajectory compared to the manikin used, a measurement has been taken from the manikin. It consists of following the sacrum shape. Both trajectories have been compared and are showed on Fig. 2.

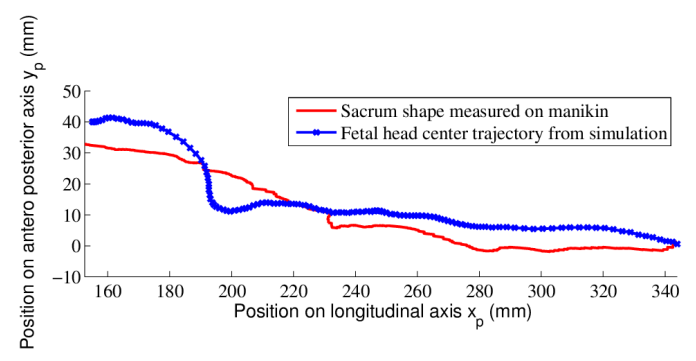

Fig. 2: Comparison between simulated trajectory of the fetal head center and the shape of sacrum measured on a manikin

Fig. 2 shows that the fetal head travels around $200 \mathrm{~mm}$ along the longitudinal axis and around $30 \mathrm{~mm}$ along the antero-posterior axis (respectively $\mathbf{x}_{p}$ axis and $\mathbf{y}_{p}$ axis on Fig. 1) during the delivery. These information were useful to estimate the stroke of our actuators.

Moreover, Fig. 2 shows that the numerical simulation respect the amplitude of the movement, but the curve trajectory due to the shape of the maternal sacrum is not actually followed. These differences stemmed from the assumptions done on the numerical models. Some improvements concerning the numerical simulations are currently under work by our partners. For a first study, it is however sufficient for the design and the first simulations. It is also noteworthy that, as only one point is tracked, only the fetal head positions are concerned. Regarding the orientations of the fetal head, they are not taken into account in this paper.

\section{DESIGN AND MODEL OF THE SIMULATOR}

\section{A. Design}

As explained before, to improve the realism of the simulator, the number of DOF of the robot should be increased. Khedar et al. concluded in 2004 that using a commercial haptic device for a childbirth simulator is not adequate and it is clearly necessary to develop a dedicated interface [10]. To improve the BirthSIM simulator realism, a 4 DOF robot has been designed. The new mechanical architecture is shown in Fig. 3.

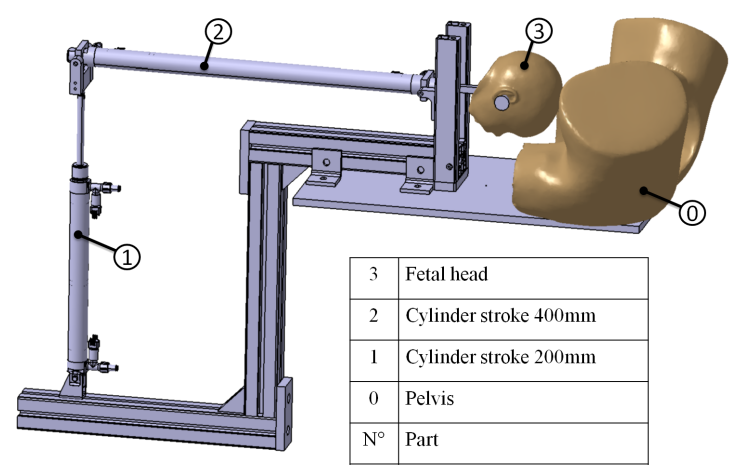

(a) $\mathrm{CAD}$

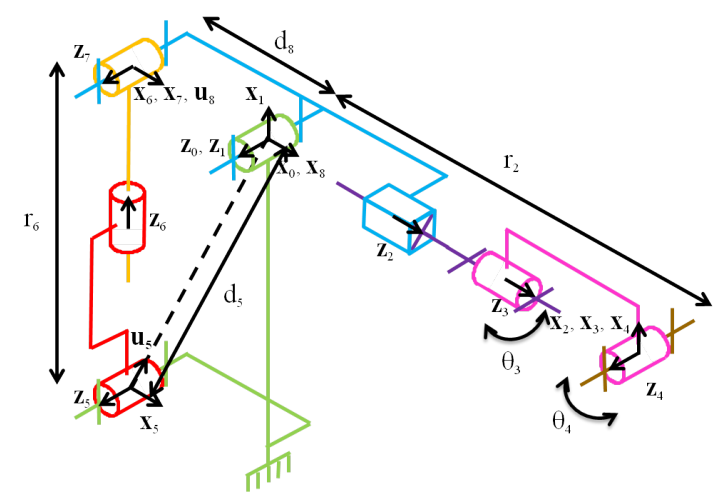

(b) Equivalent kinematic scheme

Fig. 3: Design of the simulator

Two pneumatic cylinders are used to simulate the curved trajectory of the fetal head. One of the biggest drawback of pneumatic actuators is that they have a highly non linear behavior. This drawback leads to complex control laws. Contrary to electric actuators, pneumatic actuators have however a natural compliance which is highly suitable for a realistic haptic feedback. These cylinders have been sized according to the results from [11] and [12] which showed that the 
maximal applied force to extract the fetus is about $200 \mathrm{~N}$. The stroke of these cylinders have been chosen according to the trajectory extracted from Buttin et al. [13] which is similar to the one introduced in Fig. 2.

\section{B. Model}

The geometric model of the robot have been obtained by using the modified Denavit Hartenberg paramaters and the Khalil and Kleinfiger method [14] for the robot with closed chain. These parameters are presented in the table I.

TABLE I: Geometric parameters of the BirthSIM

\begin{tabular}{|c|c|c|c|c|c|c|c|c|c|}
\hline$j$ & $a(j)$ & $\sigma$ & $\mu$ & $\mathrm{b}$ & $\mathrm{d}$ & $\mathrm{r}$ & $\gamma$ & $\alpha$ & $\theta$ \\
\hline \hline 1 & 0 & 0 & 0 & 0 & 0 & 0 & 0 & 0 & $\theta_{1}$ \\
\hline 2 & 1 & 1 & 1 & 0 & 0 & $r_{2}$ & 0 & $\frac{\pi}{2}$ & 0 \\
\hline 3 & 2 & 0 & 1 & 0 & 0 & 0 & 0 & 0 & $\theta_{3}$ \\
\hline 4 & 3 & 0 & 1 & 0 & 0 & 0 & 0 & $-\frac{\pi}{2}$ & $\theta_{4}$ \\
\hline 5 & 0 & 0 & 0 & 0 & $-d_{5}$ & 0 & $\gamma_{5}$ & 0 & $\theta_{5}$ \\
\hline 6 & 5 & 1 & 1 & 0 & 0 & $r_{6}$ & 0 & $-\frac{\pi}{2}$ & 0 \\
\hline 7 & 6 & 0 & 0 & 0 & 0 & 0 & 0 & $-\frac{\pi}{2}$ & $\theta_{7}$ \\
\hline 8 & 1 & 2 & 0 & 0 & $d_{8}$ & 0 & $-\frac{\pi}{2}$ & 0 & 0 \\
\hline
\end{tabular}

The active joint variable vector $\mathbf{q}_{a}$, passive joint variable vector $\mathbf{q}_{p}$, and cut joint variable vector $\mathbf{q}_{c}$ are respectively:

$$
\begin{aligned}
& \mathbf{q}_{a}=\left[\begin{array}{llll}
r_{2} & \theta_{3} & \theta_{4} & r_{6}
\end{array}\right]^{T} \\
& \mathbf{q}_{p}=\left[\begin{array}{ll}
\theta_{1} & \theta_{5}
\end{array}\right]^{T} \\
& \mathbf{q}_{c}=\left[\theta_{7}\right]
\end{aligned}
$$

Direct Geometric Model (DGM) is deduced from the following transformation matrix ${ }^{0} \mathbf{T}_{4}$.

$$
{ }^{0} \mathbf{T}_{4}=\left[\begin{array}{cccc}
s_{x} & n_{x} & a_{x} & P_{x} \\
s_{y} & n_{y} & a_{y} & P_{y} \\
s_{z} & n_{z} & a_{z} & P_{z} \\
0 & 0 & 0 & 1
\end{array}\right]
$$

where $s_{x}, n_{x}, a_{x}, s_{y}, n_{y}, a_{y}, s_{z}, n_{z}$ and $a_{z}$ are direction cosine matrix coefficients of the fetal head and $P_{x}, P_{y}$ and $P_{z}$ correspond to the position of the center of fetal head. Their expression are:

$$
\begin{aligned}
& s_{x}=\cos \theta_{1} \cos \theta_{3} \cos \theta_{4}-\sin \theta_{1} \sin \theta_{4} \\
& s_{y}=\cos \theta_{3} \cos \theta_{4} \sin \theta_{1}+\cos \theta_{1} \sin \theta_{4} \\
& s_{z}=\cos \theta_{4} \sin \theta_{3} \\
& n_{x}=-\cos \theta_{4} \sin \theta_{1}-\cos \theta_{1} \cos \theta_{3} \sin \theta_{4} \\
& n_{y}=\cos \theta_{1} \cos \theta_{4}-\cos \theta_{3} \sin \theta_{1} \sin \theta_{4} \\
& n_{z}=-\sin \theta_{3} \sin \theta_{4} \\
& s_{x}=-\cos \theta_{1} \sin \theta_{3} \\
& s_{y}=-\sin \theta_{1} \sin \theta_{3} \\
& s_{z}=\cos \theta_{3} \\
& P_{x}=r_{2} \sin \theta_{1} \\
& P_{y}=-r_{2} \cos \theta_{1} \\
& P_{z}=0
\end{aligned}
$$

As $\theta_{1}$ is not an active joint variable, it must be obtained by the resolution of the geometric constraint equations of the loop composed of links $0,1,5$ and 6 . This resolution gives :

$$
\theta_{1}=\gamma_{5}-\arcsin \left(\frac{r_{6}^{2}-d_{5}^{2}-d_{8}^{2}}{2 d_{5} d_{8}}\right)
$$

We assume that (4) is bijective in the working space of the robot. Moreover, with Paul method [15], the Inverse Geometric Model (IGM) is obtained.

$$
\begin{aligned}
& \theta_{3}=\operatorname{atan} 2\left(a_{x} \cos \theta_{1}-a_{y} \sin \theta_{1}, a_{z}\right) \\
& \theta_{4}=\operatorname{atan} 2\left(-s_{y} \cos \theta_{1}+s_{x} \sin \theta_{1}, n_{y} \cos \theta_{1}+n_{x} \sin \theta_{1}\right) \\
& r_{2}=-P_{y} \cos \theta_{1}+P_{x} \sin \theta_{1} \\
& r_{6}=\sqrt{d_{5}^{2}+d_{8}^{2}-2 d_{5} d_{8} \sin \left(\theta_{1}-\gamma_{5}\right)}
\end{aligned}
$$$$
\text { with } \theta_{1}=\operatorname{atan} 2\left(P_{x},-P_{y}\right) \text {. }
$$

Some of these results have been obtained with the help of Symoro+ software [16].

This IGM allows to compute a reference trajectory for each actuator from a desired end effector trajectory in position and orientation. In our case the end effector is the fetal head of the simulator.

\section{SIMULATION AND VALIDATION}

In order to validate the new design of the BirthSIM, the robot working space have been studied. Thus, from the DGM and the actuators strokes, the working space is obtained. It corresponds to the surface that the fetal head center can reached. Fig. 4 shows the borders of this surface.

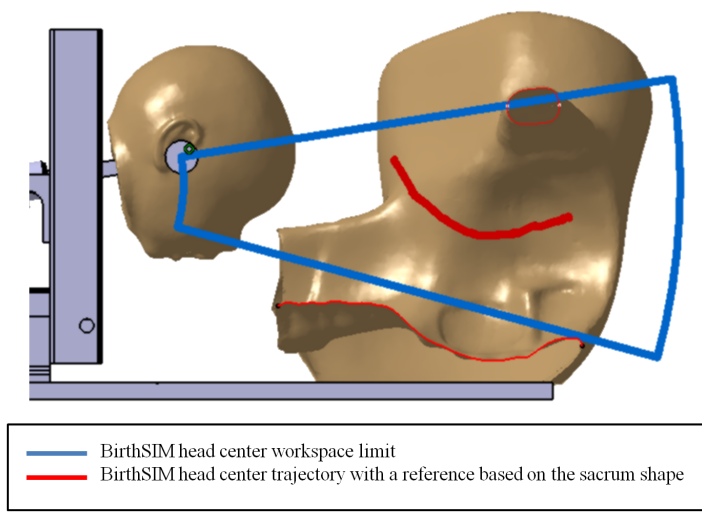

Fig. 4: Limit of the working place and a trajectory of the BirthSIM fetal head center

Indeed, as the smallest diameter of the fetal head is around $9 \mathrm{~cm}$ and that the pelvic muscles are not represented, the robot working space covers all the possible fetal head position inside the maternal pelvis. So the first goal of the new BirthSIM design, which is to increase the number of DOF to improve the realism of the fetal trajectory, is validated.

The IGM allows to obtain the displacement of each actuator from a desired trajectory of the fetal head. For instance, the trajectory plotted in Fig. 1 can be reproduced. This trajectory has two Cartesian parameters which are $P_{x}$ 
and $P_{y}$ (these parameters have been detailed in Section IIIB). By applying the IGM, the couple $\left[r_{2}, r_{6}\right]$ is obtained. In order to be more explicit, a coordinates change is necessary. Indeed $r_{2}$ and $r_{6}$ differ from the the actuator output. So two parameters are introduced $q_{1}$ and $q_{2}$, these parameters are respectively the output of the pneumatic cylinder 1 and 2 . Therefore, $q_{1}$ only depends of $r_{6}$ and $q_{2}$ only depends of $r_{2}$ and each $q_{i}$ is define on [0;stroke of cylinder $i$ ] range. Fig. 5 shows $q_{1}$ and $q_{2}$ values in order to reproduce the trajectory based on the sacrum shape. It should be noted that the time
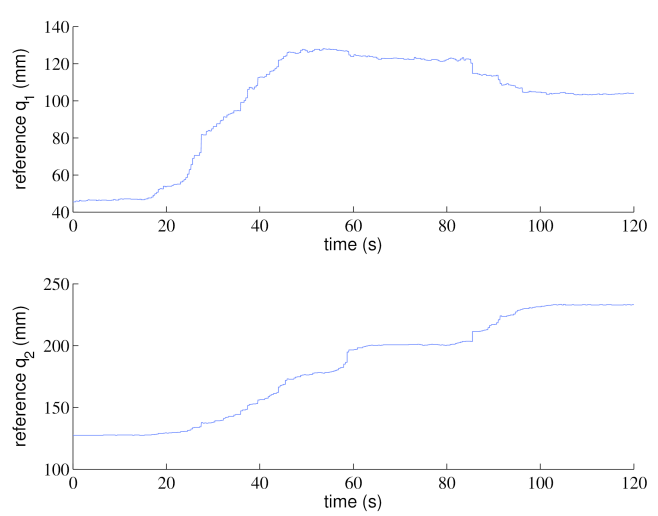

Fig. 5: $q_{1}$ and $q_{2}$ references to reproduce the fetal head trajectory based on the pelvis shape.

is not representative of a real delivery. $q_{1}$ and $q_{2}$ values have been simulated on the DGM. The result trajectory is shown on Fig. 4. This simulation also allows to validate the new mechanical design of the BirthSIM.

\section{CONCLUSION AND FUTURE WORKS}

In this paper the new mechanical architecture of the BirthSIM simulator has been presented. This haptic interface currently has 4 actuated DOF. The direct and inverse geometric models have been given and they will be used to develop control synthesis. Simulated trajectory of the fetal head during a delivery has been compared with the sacrum shape measured on a manikin. The fetal head can now have more realistic trajectories during a delivery simulation which increased the degree of realism.

For future works, more reference trajectories of position and orientation of the fetal head are needed to drive our simulator. In order to answer this need, SAGA project partners are developing numerical models of childbirth delivery.

The simulator design has thus be validated and the next step concerns the control synthesis to reproduce the dynamics of a childbirth. We plan to develop new control laws to increase the haptic feedback realism. The use of electropneumatic actuators with a stiffness control seems suitable. In [17] a control law have been developed for only one DOF system, so a scientific lock will be to complete it for multiaxes robot. To obtain this control synthesis, the kinematic and dynamic models of the BirthSIM mechanical part are necessary.

\section{ACKNOWLEDGMENT}

The authors would like to thank Romain Buttin and his advisors for sharing his work and data on on a childbirth delivery numerical model. N. H. thanks Olivier Dupuis for his explanations about the childbirth biomechanics and obstetrical science. The autors also would like to thank the ANR French National Research Agency for financing SAGA project (ANR-12-MONU-0006).

\section{REFERENCES}

[1] R. Silveira, M. T. Pham, T. Redarce, M. Betemps, and O. Dupuis, A new mechanical birth simulator: Birthsim, in Intelligent Robots and Systems, 2004 IEEE/RSJ International Conference on, vol. 4, pp. 3948-353, 2004.

[2] R. Moreau, M. T. Pham, R. Silveira, T. Redarce, X. Brun, and O. Dupuis, Design of a new instrumented forceps: Application to safe obstetrical forceps blade placement, Biomedical Engineering, IEEE Transactions on, vol. 54, no. 7, pp. 1280-1290, 2007.

[3] R. Lapeer, M. Chen, and J. Villagrana, Simulating obstetric forceps delivery in an augmented environment, in Augmented environments for Medical Imaging including Augmented Reality in Computer-aided Surgery, 2004.

[4] E. Kim, R. Allen, J. Yang, M. McDonald, W. Tam, and E. Gurewitsch, Simulating complicated human birth for research and training, in Engineering in Medicine and Biology Society, 2004. IEMBS '04. 26th Annual International Conference of the IEEE, vol. 26, pp. 2762-2766, 2004.

[5] T. Sielhorst, T. Blum, and N. Navab, "Synchronizing 3d movements for quantitative comparison and simultaneous visualization of actions, in Mixed and Augmented Reality, 2005 IEEE and ACM International Symposium on, pp. 38-47, 2005.

[6] A. Abate, G. Acampora, V. Loia, S. Ricciardi, and A. Vasilakos, A pervasive visual-haptic framework for virtual delivery training, Information Technology in Biomedicine, IEEE Transactions on, vol. 14, no. 2, pp. 326-334, 2010.

[7] S. Deering, J. Brown, J. Hodor, and A. Satin, Simulation training and resident performance of singleton vaginal breech delivery, American Journal of Obstetricians and Gynecologists, vol. 107, no. 1, pp. 86-89, 2006.

[8] C. N. des Gynécologues et Obstétriciens Fran scais (CNGOF), Engagement, tech. rep., 2006.

[9] R. Buttin, F. Zara, B. Shariat, and T. Redarce, A biomechanical model of the female reproductive system and the fetus for the realization of a childbirth virtual simulator, in Engineering in Medicine and Biology Society, 2009. EMBC 2009. Annual International Conference of the IEEE, pp. 5263-5266, Sept 2009.

[10] A. Kheddar, C. Devine, M. Brunel, C. Duriez, and O. Sibony, Preliminary design of a childbirth simulator haptic feedback, in Intelligent Robots and Systems, 2004 IEEE/RSJ International Conference on, vol. 4, pp. 3270-3275, 2004.

[11] R. Moreau, M. Pham, X. Brun, T. Redarce, and O. Dupuis, Simulation of an instrumental childbirth for the training of the forceps extraction: control algorithm and evaluation, Information Technology in Biomedicine, IEEE Transactions on, vol. 15, no. 3, pp. 364-372, 2011.

[12] K. Leslie, P. Dipasquale-Lehnerz, and M. Smith, Obstetric forceps training using visual feedback and the isometric strength testing unit, American College of Obstetricians and Gynecologists, vol. 105, no. 2, pp. 377-382, 2005.

[13] R. Buttin, F. Zara, B. Shariat, T. Redarce, and G. Grang, Biomechanical simulation of the fetal descent without imposed theoretical trajectory, Computer Methods and Programs in Biomedicine, vol. 111, no. 2, pp. $389-401,2013$.

[14] W. Khalil and E. Dombre, Modeling, Identification \& Control of Robots. Butterworth-Heinemann, 2004.

[15] R. P. Paul, Robot Manipulators: Mathematics, Programming, and Control. Cambridge, MA, USA: MIT Press, 1981.

[16] W. Khalil and D. Creusot, Symoro+: A system for the symbolic modelling of robots, Robotica, vol. 15, pp. 153-161, 1997.

[17] F. Abry, X. Brun, S. Sesmat, and E. Bideaux, Non-linear position control of a pneumatic actuator with closed-loop stiffness and damping tuning, in European Control Conference (ECC), 2013. 